\title{
The Clinical Significance of Colon Polyp Pathology in Nonalcoholic Fatty Liver Disease (NAFLD) and Its Impact on Screening Colonoscopy in Daily Practice
}

\author{
Cosmas Rinaldi A. Lesmana $\mathbb{D}^{1,2}$ Levina S. Pakasi $\mathbb{D}^{1},{ }^{2}$ Aru W. Sudoyo $\left(\mathbb{D},{ }^{2,3}\right.$ \\ Ening Krisnuhoni $\mathbb{C}^{2,4}$ and Laurentius A. Lesmana $\mathbb{1}^{2}$ \\ ${ }^{1}$ Department of Internal Medicine, Hepatobiliary Division, \\ Dr. Cipto Mangunkusumo National General Hospital, Medical Faculty, University of Indonesia, Jakarta, Indonesia \\ ${ }^{2}$ Digestive Disease and GI Oncology Centre, Medistra Hospital, Jakarta, Indonesia \\ ${ }^{3}$ Department of Internal Medicine, Hematology and Oncology Division, \\ Dr. Cipto Mangunkusumo National General Hospital, Medical Faculty, University of Indonesia, Jakarta, Indonesia \\ ${ }^{4}$ Department of Pathology Anatomy, Dr. Cipto Mangunkusumo National General Hospital, Medical Faculty, University of Indonesia, \\ Jakarta, Indonesia
}

Correspondence should be addressed to Cosmas Rinaldi A. Lesmana; medicaldr2001id@yahoo.com

Received 11 November 2020; Accepted 27 November 2020; Published 10 December 2020

Academic Editor: Branka Filipović

Copyright ( $) 2020$ Cosmas Rinaldi A. Lesmana et al. This is an open access article distributed under the Creative Commons Attribution License, which permits unrestricted use, distribution, and reproduction in any medium, provided the original work is properly cited.

\begin{abstract}
Aim. Nonalcoholic fatty liver disease (NAFLD) has been known as a risk for the presence of colon polyp and CRC development. This study was aimed to find out the clinical significance of colon polyps' pathology among NAFLD patients. Method. A retrospective database study was done in patients who underwent elective colonoscopy within one-year period in a referral private hospital, Jakarta. Subjects were adult patients who also had documented abdominal ultrasound (US). The association between NAFLD and colonic polyp was analyzed using Chi-square test with odds ratio (OR) and its corresponding 95\% confidence interval (CI). Results. A total of 138 adult patients were enrolled; 68 (51.1\%) were men. Patients' mean age was $56.8 \pm 15.3$ years old. Colon polyps were found in $49(35.5 \%)$ cases; the most common histopathology was adenoma (42.9\%). NAFLD was found in 68 (49.3\%) of patients. Colon polyps were found to be more among patients with NAFLD than in those without NAFLD (44.1\% vs. $27.1 \%$; OR: 2.119; 95\% CI: $1.040-4.318)$. Colon polyps were found in 30 (44.1\%) NAFLD patients, where 18 (26.5\%) patients had adenomatous polyp, and from this subset of patients with adenomatous polyp, $6(8.8 \%)$ patients had mild dysplasia, 8 (11.8\%) had moderate dysplasia, 1 (1.5\%) had severe dysplasia, and $3(4.4 \%)$ had adenocarcinoma. Conclusions. NAFLD is associated with increased risk of any colon polyp, regardless of the histopathological type, compared with patients without NAFLD. This finding implies the necessity to perform screening colonoscopy in patients with NAFLD in the future.
\end{abstract}

\section{Background}

Colorectal cancer (CRC) is still one of the most common cancers in the Western and Asian countries. Until now, the general recommendation for CRC screening is still based on invasive diagnostic tests, such as colonoscopy, and noninvasive tests, such as Asia-Pacific colorectal screening score (APCS) and fecal immunochemical test (FIT) [1, 2]. However, the patient's reluctance for invasive screening tests, different subgroups of patient's diversity, different screening program policies in every country, especially in developed and developing Asian countries, cost difference, and availability of diagnostic tools make a different strategy approach in the real clinical practice.

Nonalcoholic fatty liver disease (NAFLD) has been known to be associated with extrahepatic causes of death, including CRC [3]. Early studies in Asia have observed an increase of CRC risk in patients with ultrasound-diagnosed 
NAFLD compared with controls [4, 5]. The presence of NAFLD was found as an independent risk factor for colorectal adenomatous polyps in asymptomatic subjects who underwent routine colonoscopy $[6,7]$. A similar finding was also reported in Western population [8]. A systematic review involving 6263 asymptomatic subjects undergoing screening colonoscopy confirmed the association between colorectal adenoma and NAFLD [9].

A large prospective cross-sectional study in a referral private hospital in Indonesia showed that NAFLD is a common finding in unselected adult patients who underwent routine medical check-up. Several risk factors were confirmed as independent risk factors for NAFLD, that is, obesity, male gender, age of more than 35 years, high triglyceride, low high-density lipoprotein (HDL) cholesterol, and high serum alanine aminotransferase levels [10]. Notwithstanding, retrospective data analysis study of a large sample size in the same private hospital for colon polyps or cancer detection rate found almost similar important risk factors, that is, older age ( $\geq 50$ years old) and male gender [11].

However, until now, there is no consensus about how the CRC screening should be done in NAFLD population. Therefore, considering the increased prevalence of NAFLD in younger age, possible genetic and environment differences, different CRC molecular pathways, and different conclusion of pathologist's specimen evaluation, the author would like to highlight this issue based on the pathology finding of colon polyp in NAFLD patients.

\section{Method}

2.1. Study Design and Subjects. A retrospective database study was done in unselected patients who underwent elective colonoscopy within one-year period in Medistra Hospital, Jakarta. Data was collected on the patients' demography and colonoscopy findings including the presence of hemorrhoids, polyps, diverticula, inflammation, or tumor mass. Patients were included if they also had a transabdominal ultrasound study record, as it is the preferred first-line diagnostic imaging procedure for imaging screening of NAFLD [12]. Patients with evidence of hepatitis virus infection, autoimmune hepatitis, significant alcohol consumption, history of any other cancers, or familial adenomatous polyposis (FAP) were excluded.

2.2. Colonoscopy Procedure. Following full bowel preparation, a conventional colonoscopy procedure was performed in all patients using adult high-definition video fiber optic colonoscopy with an auxiliary water jet (CFQ160AL, Olympus, USA). Polyps were removed by snare-loop polypectomy at the bottom of the stalk or by biopsy forceps. Flat or sessile polyps were removed by performing a submucosal lifting technique with the injection of saline solution into the submucosal layer. Polypectomy was not performed in patients with known absolute contraindications such as anticoagulant therapy and bleeding disorders.
2.3. Histopathological Diagnosis. Biopsy specimens were evaluated and classified according to the World Health Organization (WHO) Classification by two board-certified pathologists who were blinded to clinical data. High-grade dysplasia or in situ carcinoma is defined by the considerable loss of nuclear polarity with irregular glandular architecture with no involvement beyond the muscularis mucosa. Submucosal invasive early colorectal cancer was defined as malignant lesions that invade the submucous layer, while cancer was defined as invasion of malignant cells beyond the muscularis mucosa.

2.4. Statistical Analysis. Patients' demography was presented descriptively. Categorical data were compared using the Chisquare test. A $p$ value of less than 0.05 was considered significant. Risk for polyp development in patients with NAFLD compared with patients without NAFLD was expressed as odds ratio (OR) and its corresponding 95\% confidence interval (CI). Median difference was assessed using Mann-Whitney $U$ test for skewed data. Statistical analysis was done using the SPSS software version 19.0 (SPSS Inc., Chicago, Illinois, USA).

\section{Results}

Four hundred and fourteen patients underwent elective colonoscopy during the study period; however, only 138 patients had documented of transabdominal ultrasound results. Seventy-two patients (52.2\%) were men and their mean age was $56.8 \pm 15.3$ years old. NAFLD was diagnosed in $68(49.3 \%)$ of patients. Colon polyps were found in 49 (35.5\%) patients (Table 1). The most common histopathology was adenoma and mostly having a mild dysplasia (Table 2).

Colon polyps were found among patients with NAFLD more than those without NAFLD $(44.1 \%$ vs. $27.1 \%$; $p=0.037$ ). The presence of NAFLD was associated with 2.1 times increased risk of the presence of colon polyp (Table 3 ). The median age of patients with polyp tended to be higher than that of patients without polyp (59 vs. 56 years; $p=0.639$, Mann-Whitney $U$ test). Colon polyp was found in $30(44.1 \%)$ NAFLD patients, where 18 (26.5\%) patients had adenomatous polyp, and from this subset of patients with adenomatous polyp, $6(8.8 \%)$ patients had mild dysplasia, $8(11.8 \%)$ had moderate dysplasia, 1 (1.5\%) had severe dysplasia, and $3(4.4 \%)$ had adenocarcinoma (Table 4$)$. Based on the polyps' location, $10(33.3 \%)$ patients had a right-sided polyp, and $20(66.7 \%)$ patients had a left-sided polyp (Table 5).

Based on dysplasia's type, there was no statistically significant difference between the presence of mild and moderate-to-severe grade of dysplasia in NAFLD patients (Table 6).

\section{Discussion}

The impact of colon polyps' pathology in NAFLD patients as a prevention for CRC development has not been well documented. Our current study in Indonesia, which 
TABLE 1: Characteristics of the study subjects $(N=138)$.

\begin{tabular}{lr}
\hline Variable & $n(\%)$ \\
\hline Age group & $43(31.2)$ \\
$<50$ years & $95(68.8)$ \\
$\quad>50$ years & $72(52.2)$ \\
\hline Gender & $66(47.8)$ \\
$\quad$ Male & \\
Female & $68(49.3)$ \\
\hline Presence of NAFLD & $70(50.7)$ \\
Yes & $62(91.2)$ \\
No & $5(7.3)$ \\
\hline Degree of NAFLD $(n=68)$ & $1(1.5)$ \\
Mild & $49(35.5)$ \\
Moderate & $89(64.5)$ \\
Severe & \\
\hline Presence of polyp & \\
Yes & \\
No &
\end{tabular}

TABLe 2: Characteristics of the polyps $(n=49)$.

\begin{tabular}{lc}
\hline Characteristic & $n(\%)$ \\
\hline Side of polyp $(n=49)$ & $14(28.6)$ \\
Right & $35(71.4)$ \\
Left & $21(42.9)$ \\
\hline Histopathology of polyp $(n=49)$ & $7(14.3)$ \\
Tubular adenoma & $4(8.2)$ \\
Inflammatory polyp & $3(6.1)$ \\
Serrated adenoma & $2(4.1)$ \\
Tubulovillous adenoma & $6(12.2)$ \\
Hyperplastic polyp & $6(12.2)$ \\
Adenocarcinoma & \\
Not biopsied & $16(47.1)$ \\
\hline Grade of dysplasia $(n=34)$ & $10(7.2)$ \\
Mild & $2(1.4)$ \\
Moderate & $6(4.3)$ \\
High & \\
Malignant &
\end{tabular}

TABLe 3: Associations among clinical variables and colon polyps $(n=138)$.

\begin{tabular}{|c|c|c|c|c|c|}
\hline Variable & Polyp (+) & Polyp (-) & $p$ value & OR & $95 \% \mathrm{CI}$ \\
\hline \multicolumn{6}{|l|}{ Gender } \\
\hline Male & $27(37.5)$ & $45(62.5)$ & 0.609 & 1.200 & $0.596-2.416$ \\
\hline Female & $22(33.3)$ & $44(66.7)$ & & & \\
\hline \multicolumn{6}{|l|}{ Age group } \\
\hline$\leq 50$ years & $13(30.2)$ & $30(69.8)$ & 0.384 & 0.710 & $0.328-1.536$ \\
\hline$>50$ years & $36(37.9)$ & $59(62.1)$ & & & \\
\hline \multicolumn{6}{|l|}{ NAFLD } \\
\hline Yes & $30(44.1)$ & $38(55.9)$ & 0.037 & 2.119 & $1.040-4.318$ \\
\hline No & $19(27.1)$ & $51(72.9)$ & & & \\
\hline
\end{tabular}

represents the biggest Southeast Asian country, is the first report showing an association between NAFLD and colorectal polyp. Generally, there are two responsible important factors known as the cause of the CRC development, such as genetic predisposition (FAP and HNPCC) and environmental factors; however, sporadic CRC has been noted as the most common pathway in CRC development [13]. Sporadic pathway is usually based on chromosomal instability (CIN) 
TABLE 4: Association between NAFLD and polyp type $(n=43)$.

\begin{tabular}{lccccc}
\hline NAFLD & Adenoma & Nonadenoma & $p$ value & OR & $95 \%$ CI \\
\hline Yes & $18(75.0)$ & $6(25.0)$ & $0.708^{*}$ & 0.563 & $0.120-2.626$ \\
No & $16(84.2)$ & $3(15.8)$ & & \\
\hline
\end{tabular}

${ }^{*}$ Fisher's exact test.

TABle 5: Association between NAFLD and polyps' location $(n=49)$.

\begin{tabular}{|c|c|c|c|c|c|}
\hline NAFLD & Right-sided polyp & Left-sided polyp & $p$ value & OR & 95\% CI \\
\hline Yes & $10(33.3)$ & $20(66.7)$ & 0.354 & 1.875 & $0.491-7.153$ \\
\hline No & $4(21.1)$ & $15(78.9)$ & & & \\
\hline
\end{tabular}

TABLE 6: Association between NAFLD and degree of adenoma dysplasia $(n=34)$.

\begin{tabular}{lcccrr}
\hline NAFLD & Mild dysplasia & Higher dysplasia* & $p$ value & OR & $95 \%$ CI \\
\hline Yes & $12(66.7)$ & $6(33.3)$ & 0.089 & 3.333 & $0.815-13.637$ \\
No & $6(37.5)$ & $10(62.5)$ & & \\
\hline
\end{tabular}

*Including moderate dysplasia, severe dysplasia, and malignant carcinoma.

or microsatellite instability (MSI). This pathway contributes to $\mathrm{CRC}$ development in patients with a median age of 70-75 years old. However, molecular CRC study in Indonesia showed that sporadic CRC was found in younger patients (40 years old or less) without any clear family history [14]. There has been an analysis about association between physical inactivity and CRC development [15]. Obesity is also considered as an independent risk factor for CRC development [16]. Our large prospective study has shown the significant association between high BMI and presence of NAFLD [11].

However, patients with NAFLD harbored a twofold increased risk of colon polyp, regardless of the histopathology findings. In comparison, other studies reported an increased risk of adenomatous polyp, with an OR ranging from 1.28 in patients with NAFLD [7] to 4.89 in patients with NASH, using multivariate analyses [6]. A recent metaanalysis demonstrated that NAFLD was associated with a high risk of colorectal adenoma and the number of polyps, but not with its location, size, and advanced nature [17]. Furthermore, a recent study found that advanced liver fibrosis significantly increases the risk of colorectal adenoma, advanced adenoma, and multiple adenomas [18].

In this study, colon polyps were found in $44.1 \%$ of NAFLD patients. Based on pathology point of view, adenoma (tubular and tubulovillous) and serrated adenoma were found in $50 \%$ of the patients with polyp. However, only $26.5 \%$ NAFLD patients had an adenomatous polyp. It is well known that adenomatous polyp is the most important polyp for CRC development. However, conventional adenomas (tubular, villous, and tubulovillous) are not the only precursor lesions of CRC. It is now recognized that CRC is not a single disease but more represents a constellation of heterogeneous subtypes that develop from different pathways [19]. Interestingly, our study also revealed early malignant transformation of the colon polyp in three NAFLD patients.
The current WHO classification lists several precursor lesions of $\mathrm{CRC}$, that is, adenomas, serrated lesions, chronic inflammatory bowed disease, and hamartomatous polyps (juvenile and Peutz-Jeghers polyps) [20]. Serrated lesions are a heterogeneous group that includes hyperplastic polyp, sessile serrated adenoma, and traditional serrated adenoma [21]. The earliest lesion in this group is nondysplastic aberrant crypt foci, which are considered as the precursor lesions of hyperplastic polyps [22]. Inflammatory polyps are generally nonneoplastic and are often related to inflammatory bowed disease or ischemic colitis [23]. However, we still included these polyps since their association with NAFLD has not been well established.

Since NAFLD and colon polyps share similar risk factors, patients with NAFLD might be targeted for CRC screening. Although most colon polyps are usually found in older age and elderly patients, about $25 \%$ of patients under 50 years old in our study presented with colon polyps. Considering that regular screening colonoscopy has been recommended for people aged 50 years and older [24], this finding raises the big question of whether colonoscopy should be done earlier, especially in patients with NAFLD. The recent guidelines from the American Cancer Society recommend initiating screening for CRC at the age of 45 years for all average-risk adults [25]. The recommendation to lower the starting age of screening is based on limited empirical data related to outcomes in average-risk individuals in this age group since the previous recommendation to screen at 50 years old has been largely based on expert opinion. However, the averagerisk individuals were meant for persons without a history of adenomatous polyp or colorectal cancer or a family tendency of colorectal cancer.

This study has limitations due to retrospective database study. First, it still cannot establish a cause-and-effect relationship and cannot confirm that NAFLD is an independent risk factor of colorectal adenoma. However, these 
findings have given a new insight into the prevention of CRC development in NAFLD patients. Second, the metabolic risk factors are not analyzed in this study. However, it is already well known that NAFLD is strongly related to metabolic risk factors. A larger and more comprehensive study addressing metabolic factors and colorectal polyps' pathology is needed to find stronger recommendation about screening colonoscopy in every NAFLD patients in clinical practice.

\section{Conclusions}

NAFLD is associated with increased risk of any colorectal polyp, regardless of the histopathological type, compared with patients without NAFLD. This finding implies the necessity to perform screening colonoscopy in patients with NAFLD. Further study is needed to assess other risk factors and patients' eligibility to undergo full colonoscopy when NAFLD is found during routine medical check-up.

\section{Data Availability}

The database belongs to the Endoscopy Unit of Medistra Hospital, Jakarta.

\section{Conflicts of Interest}

The authors declare that they have no conflicts of interest.

\section{References}

[1] J. J. Y. Sung, S. C. Ng, F. K. L. Chan, H. M. Chiu, H. S. Kim, and T. Matsuda, "An updated Asia pacific consensus recommendations on colorectal cancer screening," Gut, vol. 64, no. 1, pp. 1210-1232, 2015.

[2] X. He, S. Yuan, W. Li et al., "Improvement of Asia-Pacific colorectal screening score and evaluation of its use combined with fecal immunochemical test," BMC Gastroenterology, vol. 19, no. 1, p. 226, 2019.

[3] E. Vanni, A. Marengo, L. Mezzabotta, and E. Bugianesi, "Systemic complications of nonalcoholic fatty liver disease: when the liver is not an innocent bystander," Seminars in Liver Disease, vol. 35, no. 3, pp. 236-249, 2015.

[4] Y. I. Lee, Y.-S. Lim, and H. S. Park, "Colorectal neoplasms in relation to non-alcoholic fatty liver disease in Korean women: a retrospective cohort study," Journal of Gastroenterology and Hepatology, vol. 27, no. 1, pp. 91-95, 2012.

[5] V. W.-S. Wong, G. L.-H. Wong, S. W.-C. Tsang et al., "High prevalence of colorectal neoplasm in patients with non-alcoholic steatohepatitis," Gut, vol. 60, no. 6, pp. 829-836, 2011.

[6] S. T. Hwang, Y. K. Cho, J. H. Park et al., "Relationship of nonalcoholic fatty liver disease to colorectal adenomatous polyps," Journal of Gastroenterology and Hepatology, vol. 25, no. 3, pp. 562-567, 2010.

[7] K.-W. Huang, H.-B. Leu, Y.-J. Wang et al., "Patients with nonalcoholic fatty liver disease have higher risk of colorectal adenoma after negative baseline colonoscopy," Colorectal Disease, vol. 15, no. 7, pp. 830-835, 2013.

[8] A. Stadlmayr, E. Aigner, B. Steger et al., "Nonalcoholic fatty liver disease: an independent risk factor for colorectal neoplasia," Journal of Internal Medicine, vol. 270, no. 1, pp. 41-49, 2011.
[9] H. Shen, S. Lipka, A. Kumar, and P. Mustacchia, "Association between nonalcoholic fatty liver disease and colorectal adenoma: a systemic review and meta-analysis," Journal of Gastrointestinal Oncology, vol. 5, no. 6, pp. 440-446, 2014.

[10] C. Lesmana, L. Pakasi, S. Inggriani, M. Aidawati, and L. Lesmana, "Development of non-alcoholic fatty liver disease scoring system among adult medical check-up patients: a large cross-sectional and prospective validation study," Diabetes, Metabolic Syndrome and Obesity: Targets and Therapy, vol. 8, pp. 213-218, 2015.

[11] A. W. Sudoyo, C. R. A. Lesmana, E. Krisnuhoni, L. S. Pakasi, L. Cahyadinata, and L. A. Lesmana, "Detection rate of colorectal adenoma or cancer in unselected colonoscopy patients: Indonesian experience in a private hospital," Asian Pacific Journal of Cancer Prevention, vol. 15, no. 22, pp. 9801-9804, 2014.

[12] European Association for the Study of the Liver (EASL), European Association for the Study of Diabetes (EASD), and European Association for the Study of Obesity (EASO), "EASL-EASD-EASO clinical practice guidelines for the management of non-alcoholic fatty liver disease," Journal of Hepatology, vol. 64, no. 6, pp. 1388-1402, 2016.

[13] M. Ponz de Leon and A. Percesepe, "Pathogenesis of colorectal cancer," Digestive and Liver Disease, vol. 32, no. 9, pp. 807-821, 2000.

[14] M. Abdullah, A. W. Sudoyo, A. R. Utomo, A. Fauzi, and A. A. Rani, "Molecular profile of colorectal cancer in Indonesia: is there another pathway?" Gastroenterol Hepatol Bed Bench, vol. 5, no. 2, pp. 71-78, 2012.

[15] I. Min Lee, E. J. Shiroma, F. Lobelo, P. Puska, S. N. Blair, and P. T. Katzmarzyk, "Effect of physical inactivity on major noncommunicable diseases worldwide: an analysis of burden of disease and life expectancy," Lancet, vol. 380, no. 9838, pp. 219-229, 2102.

[16] M. Bardou, A. N. Barkun, and M. Martel, "Obesity and colorectal cancer," Gut, vol. 62, no. 6, pp. 933-947, 2013.

[17] W. Ding, J. Fan, and J. Qin, "Association between nonalcoholic fatty liver disease and colorectal adenoma: a systematic review and meta-analysis," International Journal of Clinical and Experimental Medicine, vol. 8, no. 1, pp. 322-333, 2015.

[18] M. C. Kim, J. G. Park, B. I. Jang, H. J. Lee, and W. K. Lee, "Liver fibrosis is associated with risk for colorectal adenoma in patients with nonalcoholic fatty liver disease," Medicine, vol. 98, p. 6, Article ID e14139, 2019.

[19] C. Langner, "Serrated and non-serrated precursor lesions of colorectal cancer," Digestive Diseases, vol. 33, no. 1, pp. 28-37, 2015.

[20] S. R. Hamilton, F. T. Bosman, P. Boffetta, M. Ilyas, H. Morreau, and S. I. Nakamura, "Carcinoma of the colon and rectum," in WHO Classification of Tumours of the Digestive System, F. T. Bosman, F. Carneiro, R. H. Hruban, and N. D. Theise, Eds., pp. 134-146, IARC, Lyon, France, 4th edition, 2010.

[21] D. C. Snover, D. J. Ahnen, R. W. Burt, and R. D. Odze, "Serrated polyps of the colon and rectum and serrated polyposis," in WHO Classification of Tumours of the Digestive System, F. T. Bosman, F. Carneiro, R. H. Hruban, and N. D. Theise, Eds., pp. 160-165, IARC, Lyon, France, 4th edition, 2010.

[22] R. Lambert, S. e. Kudo, M. Vieth et al., "Pragmatic classification of superficial neoplastic colorectal lesions," Gastrointestinal Endoscopy, vol. 70, no. 6, pp. 1182-1199, 2009.

[23] A. Dunn, "Inflammatory polyp of colon. PathologyOutlines.com website," 2019, http://www.pathologyoutlines. com/topic/colontumorinflammatory.html. 
[24] B. Levin, D. A. Lieberman, B. McFarland et al., "Screening and surveillance for the early detection of colorectal cancer and adenomatous polyps, 2008: a joint guideline from the American cancer society, the US multi-society task force on colorectal cancer, and the American college of radiology," CA: A Cancer Journal for Clinicians, vol. 58, no. 3, pp. 130-160, 2008.

[25] A. M. D. Wolf, E. T. H. Fontham, T. R. Church et al., "Colorectal cancer screening for average-risk adults: 2018 guideline update from the American Cancer Society," CA: $A$ Cancer Journal for Clinicians, vol. 68, no. 4, pp. 250-281, 2018. 